\title{
Defining Life: The Virus Viewpoint
}

\author{
Patrick Forterre
}

Received: 27 January 2009 / Accepted: 15 May 2009 /

Published online: 3 March 2010

(C) The Author(s) 2010. This article is published with open access at Springerlink.com

\begin{abstract}
Are viruses alive? Until very recently, answering this question was often negative and viruses were not considered in discussions on the origin and definition of life. This situation is rapidly changing, following several discoveries that have modified our vision of viruses. It has been recognized that viruses have played (and still play) a major innovative role in the evolution of cellular organisms. New definitions of viruses have been proposed and their position in the universal tree of life is actively discussed. Viruses are no more confused with their virions, but can be viewed as complex living entities that transform the infected cell into a novel organism - the virus - producing virions. I suggest here to define life (an historical process) as the mode of existence of ribosome encoding organisms (cells) and capsid encoding organisms (viruses) and their ancestors. I propose to define an organism as an ensemble of integrated organs (molecular or cellular) producing individuals evolving through natural selection. The origin of life on our planet would correspond to the establishment of the first organism corresponding to this definition.
\end{abstract}

Keywords Archaea $\cdot$ Evolution $\cdot$ Mimivirus $\cdot$ Origin of life $\cdot$ Virus

\section{Introduction}

What is life? This question, asked by Schrödinger sixty years ago (Schrödinger 1944), is still on the agenda. When Crick claimed that he and Watson had discovered "the secret of life", he suggested that "life is DNA", the aperiodic crystal wisely predicted by Schrödinger a few years before the discovery of the double-helix. Since then, reductionists and holists have opposed themselves in giving priority either to the genetic material, or the metabolic network to define life. Many attempts have been made to find a definition of life that could be operational not only for terrestrial life, but for any form of "life" present in the universe, a definition that could help us to recognize a bona fide extraterrestrial "life" if we encounter

P. Forterre $(\triangle)$

CNRS UMR 8621 Institut de Génétique et Microbiologie, Univ Paris-Sud, 91405 Orsay Cedex,

France and Institut Pasteur, 25 rue du Docteur Roux, 75015 Paris, France

e-mail: forterre@pasteur.fr 
it one day. In my opinion, such a definition is by essence biased by an idealist prejudice, reminiscent of Plato and Socrates' ideas. It seems to imply that life is an ideal form, concrete examples of life being various "shadows" of this ideal. I will adopt here the view that, up to now, life is only a terrestrial phenomenon, a characteristic of terrestrial "living organisms". In fact, there is no life without living organisms and all presently known living organisms are thriving on planet Earth. If one day we hopefully meet friends from another world, it will then be possible to define "life" in term of the common properties shared by organisms from both planets.

For the moment, the only materialistic way to define life is to start from the objects that exhibit this extraordinary property: being alive (or having been alive, once such objects are dead). In that sense, the question, "are viruses alive?" is clearly at the heart of the debate. The answers to this question have varied in time, depending of our knowledge about viruses and our definition of life. Over the last decades, the answer has been often negative and viruses have been usually relegated to the periphery of the living world, being mainly considered as «dangerous» curiosities. They have been considered as by-products of cellular life, having probably originated as escaped genes from cellular organisms. However, this situation is rapidly changing, following several discoveries made either by chance or by the effort of a few pioneers, and general advances in molecular biology (including the outcome of the genomic and post-genomic era) that have recently contributed to revise the position of viruses in the living world. Times are changing and viruses, once only considered as side-products of cellular evolution, are now at the center of many debates on the early evolution of life on our planet (Forterre 2002, 2005, 2006a, b; Brosius 2003; Bamford 2003; Bamford et al. 2006; Claverie 2006; Koonin et al. 2006; Ryan 2007; Raoult and Forterre 2008).

\section{Viral Particles Are the Most Abundant Biological Entities in the Biosphere}

It has been realized quite recently that viral particles are by far the most abundant biological entities on our planet (Suttle 2007). Indeed, they are ten times more abundant than bacterial cells in the upper ocean. This has been deduced in the nineties from examination of water samples by electron microscopy or epifluorescence optical microscopy. More recently, the abundance and diversity of viruses has been confirmed by the abundance and diversity of their genes in metagenomic studies (Edwards and Rohwer 2005). The fact that viruses are more abundant than their targets is not surprising, since every single cellular species is infected by many diverse viral species (as we know very well from the case of our own species, Homo sapiens) and the infection of a single cell always produces a high number of viral particles. However, the data have impressed biologists and contributed to a renewal of interest in virus research. The ecology of viruses, their roles in major geochemical cycles, and in controlling the diversity of population are now active research fields (Suttle 2007).

\section{Surprising Diversity in the Morphology of Viral Particles}

Our initial view was that of a curious but monotonous world. Viruses (confused with viral particles, see below) were essentially either small spheres (sometimes with spikes as in TV cartoons featuring the AIDS virus), or strange Lunar exploratory module (LEM) with a head, a tail, and sometimes legs (as in the case of the T4 bacteriophage and related myoviridae). Specialists (virologists) were aware of the existence of filamentous viral 
particles, or pleomorphic types of capsids (as in the case of vaccinia or poxviruses), but these were considered as exceptions. This has changed now, with the discovery, during the last two decades, that viruses infecting hyperthermophilic archaea (members of the third domain of life, see below) produce viral particles with a morphology that is completely different from the classical head and tailed structure of bacteriophages (Prangishvili et al. 2006). Some of their virions are either flexible or rigid filaments that superficially resemble those of viruses infecting bacteria or eukarya, but they form clearly distinct families (for instance, they are all double-stranded DNA viruses, whereas eukaryotic filamentous viruses are all RNA viruses). Other viral particles show morphotypes previously never seen in the viral world, such as lemon-shaped, or bottle-like structures. The most spectacular example is the virus ATV (Acidianus-Tailed-Virus) whose virion undergoes the first known case of extra-cellular development (Häring et al. 2005). The virions produced by ATV infected cells are lemon-shaped particles that can be stored for months at room temperature without any change in their morphology. However, as soon as there are incubated at high temperature (above $70^{\circ} \mathrm{C}$ ) they undergo a drastic structural reorganization, with the formation of two long tails at opposite ends of the central body (Häring et al. 2005).

\section{A New Virus Classification Inferred from the Three Domains Concept}

The unique archaeal viruses, isolated from terrestrial hot springs and infecting organisms living at temperatures between 79 and $105^{\circ} \mathrm{C}$, are not just mere curiosities. Their discovery has led to revise the classification of viruses and their relation to cellular organisms. Traditionally, viruses have been classified according to the prokaryote/eukaryote dichotomy. Historically, this binary division of the living world was proposed in 1962 by Stanier and Van Niels, following the discovery of major structural differences between bacterial cells and those of animals, fungi, plants and protists (for an historical account of this proposal and its epistemological consequences, see Sapp 2005, 2006). In bacterial cells, the genetic material (DNA) is present within the cytoplasm, being directly in contact with ribosomes, where messenger RNAs are translated into proteins. In contrast, in the cells of animals, fungi, plants and protists, the genetic material is located within a "nucleus", being separated from the cytoplasm by a nuclear membrane. Cells with a nucleus have been called eukaryotes (true nucleus) whereas cells without nucleus have been called prokaryotes (meaning before the nucleus) suggesting that they predated eukaryotes. This proposal was accepted with enthusiasm by cell biologists, but also by the pioneers of the molecular biology revolution, as a novel concept with an explanatory power much greater that older classifications favored by botanists or zoologists, such as the five kingdoms of Whittaker. Unfortunately, the concept of prokaryote had a very negative effect on virology by splitting the viral world between viruses infecting prokaryotes (bacteriophages) and viruses infecting eukaryotes (simply called viruses). It was concluded from this dichotomy that these two viral categories had different origins, bacteriophages having originated from bacterial genomes (or plasmids) and viruses from eukaryotic genomes (for instance, retroviruses from retro-elements).

However, in contradiction with this hypothesis, most viral encoded proteins, especially those involved in the replication of viral genomes, have no specific relationships with those of their hosts (Forterre 1992, 1999; Villarreal and DeFilippis 2000; Filée et al. 2002, 2003; Miller et al. 2003; Forterre et al. 2007). In contrast, viruses infecting very different hosts and producing virions with various morphologies sometimes encode similar proteins that have no homologue in the cellular world (Forterre 1999, 2005, 2006b; Koonin et al. 2006). 
The importance of these viral specific proteins (viral hallmark proteins, sensu Koonin et al. 2006) was underestimated for a long time. Since viruses were supposed to have originated from cells, the existence of real viral genes was denied (all viral genes were supposed to have originated from cells). In contrast, genomic data have shown that the huge majority of viral genes have no cellular homologues, indicating that viral genes represent a unique pool of genetic diversity.

Surprisingly, the prokaryotic concept, proposed in 1962, still functions as a paradigm for most biologists, more than 30 years after it was shown to be wrong in 1977, thanks to the work of Carl Woese and colleagues (sometimes referred to the Urbana School) (Pace 2006). In the seventies, Carl Woese applied the techniques and concepts stemming from the molecular biology revolution of the sixties to decipher the parental relationships between all living organisms. He finally demonstrated that the division of the living world between prokaryotes and eukaryotes was misleading in term of natural classification (Woese and Fox 1977). He showed that a group of organisms previously considered to be bacteria, according to their "prokaryotic phenotype" (they have no nucleus) was in fact no more related to bacteria than to eukaryotes in terms of their ribosomes (more precisely their ribosomal RNA). Although all ribosomes (the cellular organelles that synthesize proteins) are homologous in the living world, there are three versions of them. Woese and Fox concluded that living organisms should therefore be divided into three primary lineages, originally called eubacteria, archaebacteria and eukaryotes (Woese and Fox 1977). Later on, Woese and colleagues proposed to replace this nomenclature by a new one: bacteria, archaea and eukarya, to prevent further confusion between the two prokaryotic domains (archaea are not "strange" or "old" bacteria", but a domain with equal taxonomic status compared to bacteria and eukarya) (Woese et al. 1990). This trinity concept has now been corroborated by comparative biochemistry and comparative genomics. Amazingly, although archaea superficially resemble bacteria when they are examined under the microscope, they are much more similar to eukarya when they are analyzed at the molecular level (Forterre et al. 2002, for recent monographies on archaea, see ref. Cavicchioli 2007; Garrett and Klenk 2007). For example, there are 33 ribosomal proteins that are common to archaeal and eukaryotic ribosomes but are absent in bacteria (Lecompte et al. 2002).

The discovery of unique viruses infecting archaea also corroborates the three domains concept from the virus perspective. Indeed, most viruses infecting archaea have nothing in common with those infecting bacteria, although they are still considered as "bacteriophages" by many virologists, just because archaea and bacteria are both prokaryotes (without nucleus). A first step in a natural classification of viruses was thus to get rid of the dichotomy between bacteriophages and viruses, and to superimpose a viral trichotomy to the cellular trichotomy. David Prangishvili and myself have thus suggested to classify viruses into three categories, archaeoviruses, bacterioviruses and eukaryoviruses (Forterre and Prangishvili 2009).

\section{Viruses Are Ancient and Have Played a Major Role in Biological Evolution}

The last common ancestor of archaea, bacteria and eukarya is today usually called LUCA (the Last Universal Common Ancestor, or the Last Universal Cellular Ancestor). The ubiquitous existence of viruses infecting members of the three cellular domains strongly suggests that the cellular lineage of LUCA and the other cellular lineages living at that time were already victims of viral attacks. In fact, it is likely that viruses originated before LUCA, when cells still had genomes made of RNA and not DNA (DNA, which is a 
chemically modified form of RNA, could have appeared only after the emergence of complex proteins capable of modifying RNA, see Forterre 2005 and references therein). If viruses were already present in the biosphere when LUCA was living, one would expect to find some common features between viruses that now infect members of different domains. This is precisely the case. In particular, some archaeoviruses, bacterioviruses and eukaryoviruses share homologous capsid proteins and/or ATPases for protein packaging, suggesting that they all evolved from a common virus that existed at the time of LUCA of even before (Bamford 2003; Baker et al. 2005; Bamford et al. 2006; Krupovic and Bamford 2008). Based on such homologous features of their virions (defined as the virus "self" by Dennis Bamford), it has been possible to already identify three major viral lineages that probably originated independently before the time of LUCA (Bamford et al. 2006). Viruses are therefore very ancient, and the ancestral virosphere was probably already diverse and abundant at the time of LUCA. To explain why modern viruses are clearly different from one domain to the other (as previously seen in the case of archaeal viruses) we have suggested that the three ancestral populations of cellular organisms at the origin of the modern domains have randomly selected at birth three different parts of the ancestral virosphere (Prangishvili et al. 2006). The presence of a few viruses of common origin (with similar "self") in the three selected virospheres would explain the presence of homologies between some viruses infecting different domains.

The idea that viruses are very ancient and have co-evolved with the three cellular lineages from the time of LUCA and even before has recently led to several hypotheses posing that viruses have played a major role in several critical evolutionary transitions. For instance, it has been suggested that DNA and DNA replication machineries first originated in the viral world (Forterre 1999; Villarreal and DeFilippis 2000; Forterre 2002), that virusinduced transition of cells with RNA genomes into cells with DNA genomes triggered the emergence of the three cellular domains (Forterre 2006), that the nucleus of eukaryotic cells originated from a large DNA virus (Takemura 2001; Bell 2001), or even that the selection pressure to prevent the entry of virions promoted the evolution of cell walls (Jalasvuori and Bamford 2008). All these hypotheses are not easily testable, but recent findings make them reasonable. Indeed, it has been shown that cellular proteins playing very important roles in modern organisms may have a viral origin. For instance, phylogenetic analyses have revealed that the RNA polymerase, DNA polymerase and DNA helicase that transcribe and replicate DNA in modern mitochondria were recruited from a virus that was originally integrated into the genome of the bacterium at the origin of the mitochondria (Filée and Forterre 2005). More recently, it has been shown that placentation in mammals is initiated by a protein, syncitin, encoded by a retrovirus integrated in mammalian chromosomes (De Parseval and Heidmann 2005; Prudhomme et al. 2005). There are many other examples of the role that viruses have played in recent cellular evolution (for reviews, see Ryan 2007; Brosius 2003; Villarreal 2005). Brosius wrote, for instance, that "the interaction of hosts with retroviruses, retrotransposons and retroelements is one of the eternal conflicts that drive the evolution of life" (Brosius 2003). Prangishvili and myself have recently extended his argument, concluding that the conflict between cells and viruses has been (and still is) the major engine of life evolution (Forterre and Prangishvili 2009).

\section{The Nature of Viruses}

For a long time, viruses have been defined by their virions, the viral particles produced during infection. The confusion between the virus and the virion is still apparent both in the 
media (the AIDS virus on TV is shown as a sphere with spikes - the virion) and in the scientific literature (when it is claimed that viruses are ten times more abundant than bacteria in the ocean, it is meant that viral particles are ten times more abundant). As a consequence of this confusion, viruses were first defined as simple entities (for instance with a single type of nucleic acid, as in the famous André Lwoff's definition, Lwoff 1957), without any metabolic activity. Since some virions can crystallize, viruses were considered as molecular (not cellular) entities. Many definitions of life being based on the cellular theory "Omniae cellula e cellula" (for instance, in his Nobel lecture, Anfré Lwoff wrote "an organism is constituted of cells" Lwoff 1967), viruses were not usually classified as living organisms.

The confusion between the virus and the virion was first criticized by Claudiu Bandea who considered that the intracellular phase of the virus life cycle is the ontogenetically mature phase of viruses (Bandea 1983). As Bandea wrote in a landmark paper "in this phase the virus shows the major physiological properties of other organisms: metabolism, growth, and reproduction. Therefore, life is an effective presence". The proposal of Bandea was ignored until recently, when the discovery of the giant mimivirus by Didier Raoult and his colleagues (La Scola et al. 2003; Raoult et al. 2004) focused the attention of virologists on the viral factory. Eukaryotic viruses that replicate in the cytoplasm form complex localized viral factories to replicate their genome and produce virions (Novoa et al. 2005, Miller and KrijnseLocker 2008). The viral factories of the mimivirus are spectacular and their size is similar to the size of the nucleus of the virus host, the amoebae Acanthameba polyphaga (Suzan-Monti et al. 2007). The virion of mimivirus is itself much larger than all previously known viral particles, being visible under the light microscope and its size being similar to those of small cells, such as mycoplasma. Jean-Michel Claverie pointed out that the viral factory corresponds to the real viral organism, whereas the virion corresponds to the mechanism used by the virus to spread from one cell to others and that to confuse the virion with the virus would be the same as to confuse a sperm cell with a human being (Claverie 2006).

One can wonder why the confusion between viruses and their virions became a paradigm in virology. This is probably because our modern conception of viruses was mostly elaborated following the work on "bacteriophages" performed in the fifties by the "phage group" in the USA and André Lwoff in France. Indeed, bacterioviruses did not produce viral factories and the viruses seemed to disappear (being reduced to their genomes) during the intracellular phase of their life cycle, known as the "eclipse phase". Interestingly, Lwoff wrote forty years ago that the virus transforms the entire infected cell into a viral factory (Lwoff 1967). If we consider now that the virus and the virion should not be confused, his sentence can be read: bacterioviruses (and archaeoviruses) transform the infected cell into a virion factory, i.e. into a virus! Many lytic viruses indeed trigger the degradation of the host genome. In that case, after destruction or inactivation of the cellular genome, when the viral genome is the only one that is expressed, one can really consider that the infected "cell" is no more a bacterium, but a virus with a cellular appearance. A nice example of this conversion is provided by cyano-bacterioviruses (cyanophages) that encode their own photosynthetic proteins to replace the decaying cellular ones in order to get the proper energy required for the production of virions (Bragg and Chisholm 2008 and references therein). The former cyanobacterial cell thus becomes a photosynthetic virus. We observed recently the same type of conversion in the case of a virus infecting a hyperthermophilic archaeon (Bizet et al. 2009). This virus destroys the genome of its host and produces spectacular intracellular structures that break the cell envelope to prepare the release of its virions.

If infected archaea and bacteria are indeed transformed into bona fide viruses, one can conclude that infected eukaryotic cells in which viral factories have taken control of the 
cellular machinery became viruses themselves, the viral factory being in that case the equivalent of the nucleus. By adopting this viewpoint, one should finally consider viruses as cellular organisms. They are of course a particular form of cellular organism, since they do not encode their own ribosomes and cell membranes, but borrow those from the cells in which they live.

The question, "are viruses alive?" is typically a philosophical question, meaning that it is our choice to decide if viruses are living entities or not. For a growing number of evolutionists and virologists, viruses should definitely be considered as living entities since they exhibit all features typical of terrestrial life: they are made of the same macromolecules as cells from archaea, bacteria or eukarya, and they have co-evolved with members of these three domains according to the scheme of Darwinian evolution. Amazingly, the recent discovery that the virion factory of the mimivirus can be infected by another virus (sputnick) has also been taken as an argument in favor of the living nature of viruses (only living organisms can become ill) (La Scola et al. 2008; Pearson 2008). Finally, considering viruses themselves as cellular organisms reconciles the idea that viruses are living with the classical definition of living organisms as cellular organisms (Lwoff 1967). To take into account the idea that viruses represent a bona fide form of life, Didier Raoult and myself have recently proposed to divide the living world into two major groups of organisms, ribosome encoding-organisms (the descendants of LUCA, archaea, bacteria and eukarya) and capsid-encoding organisms (the viruses) (Raoult and Forterre 2008).

\section{What is Life?}

Although the definitions of life have evolved continuously depending on the progress of our knowledge in biology, this is clearly not a scientific question, but a philosophical one. Definitions of life have always been based at a given time on the philosophical background of scientists as well as the scientific background of philosophers. As a consequence, the answer to the question, "what is life?" will always be given in a particular philosophical framework. Personally, although dialectic materialism is now out of fashion for historical and political reasons, I like the definition of life proposed in the 19th century by Frederich Engels in his posthumous book Dialectics of Nature. For Engels, "life is the mode of existence of albuminoïd bodies" (Engels 1883). At the time of Engels, it was a prescient insight to focus the definition of life on proteins (albuminoïds), considering that the real nature, diversity and role of proteins were then practically unknown. At first sights, a modern version of this definition could be: "life is the mode of existence of informational macromolecules (proteins and nucleic acids)". However, the term "albuminoïd bodies" asks for more. Albuminoïd bodies could be translated in modern time as "a physical entity based on organic molecules, molecules that are produced by living entities, let's say ... an organism". So I would give the following definition of life: 'life is the mode of existence of living organisms'. If one only considers present terrestrial life, one could conclude that "life is the mode of existence of ribosomal and capsid encoding organisms (REO and CEO)". However, we would like to reach a definition that would also include ancient terrestrial life (predecessors of modern REO and CEO), especially in the framework of discussions about the origin of life. So the question remains, what is a living organism? According to Lwoff (1967) "an organism is an integrated system of interdependent structures and functions".

I will define here a living organism as an entity formed by the functional integration of several "organs", corresponding to the structure and functions of Lwoff's definition. By analogy with multicellular organisms that are composed of several organs (skin, liver, brain 
and so on), unicellular organisms can be defined as composed of several molecular machines and/or structures (metabolic networks, ribosomes, replicons, capsid, membranes and so on). A living organism can thus be defined as: "a collection of integrated organs (molecular machines/structures) producing individuals evolving through natural selection".

The simplest viruses encode two different "organs", a replicon, allowing genome multiplication, and a capsid, i.e. a complex structure allowing not only to protect the viral genome in the extracellular space, but also involved in the entrance and exit mechanisms of virions in and out of the cell. All viruses encode sophisticated mechanisms to divert the organs of the infected cells, such that these organs become part of the viral organism during infection.

One can try to use our definition of organisms to approach the problem of the origin of life itself. Modern cells descending from LUCA and their viruses are all complex organisms, and LUCA itself has been the product of a long history (for a recent review, see Forterre and Gribaldo 2007). Life indeed already existed before the emergence of capsids and ribosomes. This is the reason why I included the ancestors of LUCA in my definition of life. At some point one should have to imagine the nature of primitive cells to include their features in our definition. The precise moment when life originated corresponds to the appearance of the first individuals formed by at least two integrated molecular organs (possibly a primitive metabolic network and a membrane) co-evolving through natural selection.

Although the definition of life is a philosophical question, the choice of a definition has a great impact in the definition of scientific programs. The definition of life proposed here implies that the goal of biology should be to explore and understand exhaustively (via combining reductionist and integrative approaches) the mode of existence of living organisms and to understand their history (evolution being the cornerstone of biology). Above all, a program to study "the origin of life" should focus on looking, theoretically and experimentally, for the mechanisms that led to the emergence of the first living organisms on our planet.

Acknowledgments I thank Michel Morange for the invitation to participate to the 2008 meeting on life definition in Paris. I am grateful to David Prangishvili, Didier Raoult and Simonetta Gribaldo for fruitful discussions.

Open Access This article is distributed under the terms of the Creative Commons Attribution Noncommercial License which permits any noncommercial use, distribution, and reproduction in any medium, provided the original author(s) and source are credited.

\section{References}

Baker ML, Jiang W, Rixon FJ et al (2005) Common ancestry of herpes viruses and tailed DNA bacteriophages. J Virol 79:14967-14970

Bamford DH (2003) Do viruses form lineages across different domains of life? Res Microbiol 154:231-236

Bamford DH, Grimes JM, Stuart DI (2006) What does structure tell us about virus evolution? Curr Opin Struct Biol 15:655-663

Bandea C (1983) J Theor Biol 105:591-602

Bell PJ (2001) Viral eukaryogenesis: was the ancestor of the nucleus a complex DNA virus? J Mol Evol $53: 251-256$

Bizet A, Karlsson EA, Ekefjärd K, Prevost MC, Forterre P, Tenaillon O, Bernander R, Prangishvili D (2009) A Unique Virus Release Mechanism in Archea. Proc Natl Acad Sci, in press

Bragg JG, Chisholm SW (2008) Modeling the fitness consequences of a cyanophage-encoded photosynthesis gene. PLoS ONE 3(10): 3550 
Brosius J (2003) The contribution of RNAs and retroposition to evolutionary novelties. Genetica 118:99-116 Cavicchioli R (2007) Archaea: molecular and cellular biology. ASM

Claverie JM (2006) Viruses take center stage in cellular evolution. Genome Biol 7:110

De Parseval N, Heidmann T (2005) Human endogenous retroviruses: from infectious elements to human genes. Cytogenet Genome Res 110:318-332

Edwards RA, Rohwer F (2005) Opinion: viral metagenomics. Nat Rev Microbiol 3:504-510

Engels F (1883) Dialectics of nature, 1-410. Wellred, London, 2006

Filée J, Forterre P (2005) Viral proteins functioning in organelles: a cryptic origin? Trends Microbiol 13:510513

Filée J, Forterre P, Sen-Li T et al (2002) Evolution of DNA polymerase families: evidences for multiple gene exchange between cellular and viral proteins. J Mol Evol 54:763-773

Filée J, Forterre P, Laurent J (2003) The role played by viruses in the evolution of their hosts: a view based on informational protein phylogenies. Res Microbiol 154:237-43

Forterre P (1992) New hypotheses about the origins of viruses, prokaryotes and eukaryotes. In: Thanh Vân JK, Mounolou JC, Schneider J, Mc Kay C (eds) Frontiers of life. Edition Frontières, Gif-sur-Yvette, pp 221-234

Forterre P (1999) Displacement of cellular proteins by functional analogues from plasmids or viruses could explain puzzling phylogenies of many DNA informational proteins. Mol Microbiol 33:457-465

Forterre P (2002) The origin of DNA genomes and DNA replication. Curr Opin Microbiol 5:525-532

Forterre P (2005) The two ages of the RNA world, and the transition to the DNA world: a story of viruses and cells. Biochimie 87:793-803

Forterre P (2006a) Three RNA cells for ribosomal lineages and three DNA viruses to replicate their genomes: a hypothesis for the origin of cellular domain. Proc Natl Acad Sci 103:3669-3674

Forterre P (2006b) The origin of viruses and their possible roles in major evolutionary transitions. Virus Res 117:5-16

Forterre P, Gribaldo S (2007) The origin of modern terrestrial life. HFSP J 1:156-168

Forterre P, Prangishvili D (2009) The great billion-year war between ribosome- and capsid-encoding organisms (cells and viruses) as the major source of evolutionary novelties. Proc NY Acad Sci, 1178:65-77

Forterre P, Brochier C, Philippe H (2002) Evolution of the Archaea. Theor Popul Biol 61:409-422

Forterre P, Gribaldo S, Gadelle D, Serre MC (2007) Origin and evolution of DNA topoisomerases. Biochimie 9:427-46

Garrett R, Klenk HP (2007) Archaea: evolution, physiology and molecular biology. Blackwell

Häring M, Vestergaard G, Rachel R, Chen L, Garrett RA, Prangishvili D (2005) Virology: independent virus development outside a host. Nature 436:1101-1102

Jalasvuori M, Bamford JKH (2008) Structural co-evolution of viruses and cells in the primordial world. Orig Life Evol Biosph 38:165-181

Koonin EV, Senkevich TG, Dolja VV (2006) The ancient Virus World and evolution of cells. Biol Direct 9:1-29

Krupovic M, Bamford DH (2008) Virus evolution: how far does the double beta-barrel viral lineage extend? Nat Rev Microbiol 6:941-948

La Scola B, Audic S, Robert C et al (2003) A giant virus in amoebae. Science 299:2033

La Scola B, Desnue C, Pagnier P et al (2008) The virophage, a unique parasite of the giant Mimivirus. Nature 455:100-104

Lecompte O, Ripp R, Thierry JC, Moras D, Poch O (2002) Comparative analysis of ribosomal proteins in complete genomes: an example of reductive evolution at the domain scale. Nucleic Acids Res 30:53825390

Lwoff A (1957) The concept of virus. J Gen Microbiol 17:239-253

Lwoff A (1967) Principles of classification and nomenclature of viruses. Nature 215:13-14

Miller S, Krijnse-Locker J (2008) Modification of intracellular membrane structures for virus replication. Nat Rev Microbiol 6:363-374

Miller ES, Kutter E, Mosig G, Arisaka F, Kunisawa T, Rüger W (2003) Bacteriophage T4 genome. Microbiol Mol Biol Rev 67:86-156

Novoa RR, Calderita G, Arranz R, Fontana J, Granzow H, Risco C (2005) Virus factories: associations of cell organelles for viral replication and morphogenesis. Biol Cell 97:147-172

Pace NR (2006) Time for change. Nature 441:289

Pearson H (2008) Virophage' suggests viruses are alive. Nature 454:677

Prangishvili D, Forterre P, Garrett RA (2006) Viruses of the Archaea: a unifying view. Nat Rev Microbiol 4:837-848

Prudhomme S, Bonnaud B, Mallet F (2005) Endogenouse retroviruses and animal reproduction. Cytogenet Genome Res 110:353-364

Raoult D, Forterre P (2008) Redefining viruses: lessons from Mimivirus. Nat Rev Microbiol 6:315-319 
Raoult D, Audic S, Robert C et al (2004) The 1.2-megabase genome sequence of Mimivirus. Science 306:1344-1350

Ryan RF (2007) Viruses as symbionts. Symbiosis 44:11-21

Sapp J (2005) The prokaryote-eukaryote dichotomy: meanings and mythology. Microbiol Mol Biol Rev 69:292-230

Sapp J (2006) Two faces of the prokaryote concept. Int Microbiol 9:163-172

Schrödinger E (1944) What is life? The physical aspect of the living cell. Cambridge University Press, Cambridge

Suttle CA (2007) Marine viruses-major players in the global ecosystem. Nat Rev Microbiol 5:801-812

Suzan-Monti M, La Scola B, Barrassi L et al (2007) Ultrastructural characterization of the giant volcano-like virus factory of Acanthamoeba polyphaga Mimivirus. PLoS ONE 2:e328

Takemura M (2001) Poxviruses and the origin of the eukaryotic nucleus. J Mol Evol 52:419-425

Villarreal LP (2005) Viruses and the evolution of life. ASM, Washington

Villarreal LP, DeFilippis VR (2000) A hypothesis for DNA viruses as the origin of eukaryotic replication proteins. J Virol 74:7079-7084

Woese CR, Fox GE (1977) Phylogenetic structure of the prokaryotic domain: the primary kingdoms. Proc Natl Acad Sci USA 74:5088-5090

Woese CR, Kandler O, Wheelis ML (1990) Towards a natural system of organisms: proposal for the domains Archae, Bacteria, and Eukarya. Proc Natl Acad Sci USA 87:4576-4579 